\title{
Evaluation of electrocardiographic ventricular repolarization parameters in stable coronary artery disease
}

\author{
Mehmet Cosgun, Emrah Erdal, Yilmaz Gunes, Isa Sincer, Asli Kurtar Mansiroglu
} Department of Cardiology, Bolu Abant Izzet Baysal University, Faculty of Medicine, Bolu, Turkey

\section{ABSTRACT}

Aim: To examine the relationship between the SYNTAX score (SS) and cardiac repolarization parameters such as cQTd and Tp-e values, and Tp-e/QT, Tp-e/JT, Tp-e/QTc, and Tp-e/JTc ratios in patients who have stable angina pectoris.

Methods: 12-lead resting electrocardiograms (ECGs) and SS of 160 patients (51 female and 109 male) undergoing coronary angiography with the pre-diagnosis of stable angina pectoris were evaluated. Patients with a SS below 22 were classified as Group 1 (low-SYNTAX), and those above 22 (high-SYNTAX) as Group 2. Forty-four patients with normal coronary angiography were included in Group 3.

Results: Mean age of the patients was 62.4 \pm 9.1 years. The heart rate, QRS, QT, cQT and JT durations between the groups were similar. In addition, relatively recent ventricular repolarization indices such as Tp-e interval and Tp-e/QT, Tp-e/JT, Tp-e/QTc, and Tp-e/JTc ratios were also not substantially different between groups.

Conclusion: Several surface ECG predictors of ventricular arrhythmias, including QTd, JT and Tp-e intervals and their ratios to QT and JT, are not significantly correlated with SYNTAX score-assessed CAD severity in patients with stable angina pectoris.

Keywords: Arrhythmia, coronary artery disease, SYNTAX score, Tp-e interval, Tp-e/QTc ratio, Tp-e/JTc ratio.

Dr. Mehmet Cosgun

Department of Cardiology, Bolu Abant Izzet Baysal University, Faculty of Medicine, Bolu, Turkey

E-mail: coskun44@gmail.com

Received: 2021-02-16 Revised: 2021-03-03

Accepted: 2021-03-08 / Published online: 2021-04-01

\section{Introduction}

The leading cause of death in developing countries is coronary artery disease (CAD) [1]. In 20-25 percent of patients, sudden cardiac death (SCD) can be the first clinical appearance of ischemic heart disease. About $75 \%$ to $80 \%$ of all SCDs are due to ischemic heart disease [2,3]. SCD is generally associated with lifethreatening ventricular arrhythmias that may cause irregular electrophysiological substrates for myocardial ischemia. It is controversial to classify high risk patients by surface electrocardiogram (ECG) measures [4,5].

In order to predict people at high risk for ventricular arrhythmias, many surface ECG markers such as $\mathrm{T}$ wave peak-to-end interval (Tp-e) and JT interval (JT), QT interval (QT) and QT dispersion (QTd) were suggested [6,7]. Another ventricular repolarization (VR) index that remains stable despite dynamic changes in the heart rate (HR) is the Tp-e/QT ratio [8,9]. The SYNTAX score (SS) was created as a combination of several validated angiographic classifications to grade the coronary anatomy in terms of the number of lesions and their functional effects, position, and complexity 
[10]. Initially the SS has been suggested as an aid to decision making for percutaneous coronary intervention rather than a predictive tool for percutaneous coronary intervention. On the other hand, its prognostic utility has been validated in various settings [10-13]. We investigated the relationship between the SS and the repolarization indices of 12-lead surface ECG in this report.

\section{Materials and methods}

Between March 2019 and January 2020, this retrospective study was performed at Bolu Abant Izzet Baysal University Faculty of Medicine Hospital. The study procedure has been approved by the Ethics Committee and written informed consent was given by each participant (Date and decision number: 2020/06). The study was conducted in accordance with the principles of the Declaration of Helsinki. All patients and the institution were informed about the study and their written consents were obtained.

For the study, a total of 171 patients who underwent coronary angiography with the prediagnosis of stable angina pectoris (SAP) were examined. Medical history of patients with percutaneous intervention (PCI) or bypass operation, coronary artery disease, antiarrhythmic substance usage, cardiac failure, serious renal failure, and chronic obstructive pulmonary disease were excluded. Seven patients were excluded from the study because of atrial fibrillation; three patients were excluded due to the left branch block of the bundle and one patient was excluded due to the right branch block of the ECG bundle. As a result, the study included 160 participants. For patients with coronary angiography lesions of 50 percent or more, the SS was computed. Patients with a SS below 22 was classified as Group 1 (low-SYNTAX) those above 22 (high-
SYNTAX) as Group 2. Group 3 included fortyfour patients with normal coronary angiography.

\section{Electrocardiography}

After 10 minutes of rest, twelve-lead ECGs were obtained with an amplitude of $10 \mathrm{~mm} / \mathrm{mV}$ and a rate of $25 \mathrm{~mm} / \mathrm{s}$ in the supine position using the available machines (Nihon Kohden Cardio fax ECG-1950 VET). ECGs were measured manually with a magnifying glass (TorQ $150 \mathrm{~mm}$ Optical Caliper LCD) by two blinded cardiologists. QT intervals were measured from the onset of the QRS complex to the end of the $T$ wave, which was described as its return to the TP baseline. JT interval was defined as the distance between the end of the QRS complex ( $\mathrm{J}$ point) and the end of the T wave. The R-R interval was calculated and used with Bazett's formula to calculate the HR and to calculate QTc and JTc [14]. The Tp-e interval was calculated as the distance between the peak and en of the $\mathrm{T}$ wave. The difference between the maximum and minimum QT interval in various leads was defined as QTd. Also ratios of Tp-e to QT, Tp-e to JT, Tp-e to QTc and Tpe to JTc were calculated. For measurements, the intraobserver and interobserver differences were less than 10 percent.

\section{Statistical analysis}

Windows Operating System Statistical Package Program SPSS 15.0 (SPSS Inc, Chicago, Illinois, USA) was used. Quantitative variables were expressed as mean \pm standard deviation (SD), median and interquartile range. To determine the discrepancies between groups we used the Anova and Student t-test for normally distributed variables and Kruskal Wallis test and the Mann-Whitney U-test for variables without normal distribution. Chi-square test was used for qualitative variables. A two-tailed $P$ value of less than 0.05 was accepted to be significant. 
Table 1. General characteristics of the study groups.

\begin{tabular}{|l|c|c|c|c|}
\hline $\begin{array}{l}\text { Baseline } \\
\text { characteristics }\end{array}$ & Group 1 (n=66) & Group 2 (n=50) & Group 3 (n=44) & P value \\
\hline Age (years) & $61.6 \pm 9.3$ & $64.7 \pm 6.8$ & $60.4 \pm 8.1$ & 0.060 \\
\hline HT (n, \%) & $38 / 60(63.3)$ & $24 / 42(57.1)$ & $27 / 44(61.4)$ & 0.821 \\
\hline DM (n, \%) & $21 / 60(35)$ & $18 / 42(42.9)$ & $12 / 44(27.3)$ & 0.317 \\
\hline HL (n, \%) & $6 / 60(10)$ & $4 / 42(9.5)$ & $6 / 44(13.6)$ & 0.791 \\
\hline Smoking (n, \%) & $31 / 60(51.7)$ & $28 / 42(66.7)$ & $12 / 44(27.3)$ & $\mathbf{0 . 0 0 1}$ \\
\hline LVEF, mm & $60.2 \pm 3.7$ & $58.6 \pm 4.1$ & $60.1 \pm 3.4$ & 0.096 \\
\hline SYNTAX score & $11.1 \pm 5.2$ & $27.1 \pm 4.2$ & & $<0.001$ \\
\hline & Median (IQR) & Median (IQR) & Median (IQR) & \\
\hline LAD, mm & $36.5(6)$ & $35.5(5)$ & $36(5)$ & 0.445 \\
\hline LVEDd, mm & $47.5(7)$ & $49(7)$ & $46(7)$ & 0.313 \\
\hline
\end{tabular}

Values are expressed as mean $\pm S D$ or median (IQR: Interquartile range) HT: Hypertension, DM: Diabetes Mellitus, HL: Hyperlipidemia, EF: Left ventricular ejection fraction, LAD: Left atrium diameter, LVEDd: Left ventricular end-diastolic diameter.

Table 2. Electrocardiographic measurements of the study groups.

\begin{tabular}{|l|c|c|c|c|}
\hline Variables & Group 1 (n=66) & Group 2 (n=50) & Group 3 (n=44) & P value \\
\hline Heart rate (bpm) & $69.4 \pm 12.8$ & $71.5 \pm 13.2$ & $69.8 \pm 11.2$ & 0.759 \\
\hline QT max. (ms) & $364.4 \pm 28.3$ & $359.4 \pm 28.8$ & $360.3 \pm 22.4$ & 0.600 \\
\hline QT min. (ms) & $346.2 \pm 28.4$ & $342.1 \pm 29.3$ & $345.2 \pm 23.4$ & 0.756 \\
\hline QT mean (ms) & $355.3 \pm 27.9$ & $350.9 \pm 28.2$ & $352.7 \pm 22.7$ & 0.716 \\
\hline QTc (ms) & $378.5 \pm 27.6$ & $378.7 \pm 28.9$ & $377.9 \pm 22.1$ & 0.990 \\
\hline Tp-e/JT & $0.326 \pm 0.04$ & $0.325 \pm 0.04$ & $0.309 \pm 0.04$ & 0.118 \\
\hline Tp-e/JTc & $0.306 \pm 0.04$ & $0.300 \pm 0.05$ & $0.287 \pm 0.4$ & 0.118 \\
\hline Tp-e/QT & $0.238 \pm 0.02$ & $0.230 \pm 0.02$ & $0.227 \pm 0.02$ & 0.131 \\
\hline Tp-e/QTc & $0.224 \pm 0.03$ & $0.213 \pm 0.03$ & $0.212 \pm 0.2$ & 0.097 \\
\hline & Median (IQR) & Median (IQR) & Median (IQR) & \\
\hline Tp-e (ms) & $85(16)$ & $80(14)$ & $81(13)$ & 0.072 \\
\hline QRS duration (ms) & $94.5(16)$ & $95(13)$ & $92(12)$ & 0.131 \\
\hline JT (ms) & $264(38)$ & $259.7(38)$ & $261.9(34)$ & 0.141 \\
\hline JTc (ms) & $281(36.7)$ & $262(49.2)$ & $283(36.5)$ & 0.392 \\
\hline QTd (ms) & $18(12.2)$ & $19.5(14)$ & $14(8)$ & 0.062 \\
\hline cQTd & $21(13)$ & $21.5(15.2)$ & $16(7.5)$ & 0.065 \\
\hline
\end{tabular}

Values are expressed as mean $\pm S D$ or median (IQR: Interquartile range). QTc: Corrected QT, QTd: QT dispersion, bpm: Beat per minute, ms: Millisecond, Tp-e: T peak and end interval, cQTd: corrected QT dispersion. 


\section{Results}

The participants mean age was $62.4 \pm 9.1$ years. There were 51 female and 109 male patients. There were no major variations between the groups in the prevalence of hypertension, diabetes mellitus and hyperlipidemia. In Group-2, smoking was substantially higher in comparison to other groups. Additionally, left atrium diameter, and ejection fraction and enddiastolic diameter of left ventricle (LV) did not vary significantly between groups (Table 1). QRS duration, HR, and QT, QTd and JT values were similar between groups. Furthermore, relatively recent repolarization indices such as values of cQTd and Tp-e, and ratios of Tp-e/JT, Tp-e/JTc, Tp-e/QT and Tp-e/QTc were not substantially different between groups (Table 2).

\section{Discussion}

In the present analysis, we observed that the length of QRS, HR, QTd, JT, Tp-e values and Tp-e/JT and Tp-e/QT ratios did not vary significantly between high-SYNTAX and lowSYNTAX and normal coronary anatomy groups.

SCD is typically triggered by ventricular arrhythmias, especially ventricular fibrillation [2,3]. Myocardial ischemia and infarction are among the most common underlying mechanisms for these life-threatening ventricular arrhythmias $[15,16]$. There is a 'border zone' between normal and hypoxic/ischemic tissues that can promote the occurrence of arrhythmias through several mechanisms such as automatic action during myocardial ischemia $[17,18]$. Shortened refractoriness and slowed conduction are the most pronounced electrophysiological impacts of myocardial ischemia [19].

The interval between the QRS complex and the end of the T wave in ECG is the product of ventricular depolarization and repolarization. Cardiac electrical changes during this phase, especially during VR, can lead to fatal ventricular arrhythmias [20]. The time from the onset of ventricular depolarization to the end of repolarization is the QT interval. The JT interval is a mere VR time component and has been suggested to be more VR specific than the QT [21,22].

It has recently been suggested that the VR dispersion corresponds to the Tp-e interval $[23,24]$. The ratio of Tp-e/QT has been hypothesized as a potential arrhythmogenesis index independent of the length of the QT interval. This relatively new VR index stay to be fixed despite alterations in HR [8,9].

It has been shown that QT, QTc and QTd values predict life-threatening ventricular arrhythmic events and SCD in CAD. Corrected QTd was increased in patients with healthy CAD and was linked to the degree and severity of coronary atherosclerosis [25]. Panikkath et al. [26] compared ECGs of SCD cases $(n=353$; mean age, 66.6 years) with living controls having CAD ( $n=342$; mean age, 64.7 years) using a case-control design. The mean Tp-e value, and QRS and QTc durations were significantly prolonged and the Tp-e/QT ratio was higher in SCD cases. After adjusting for gender, age, QRS duration, value of QTc and LV function, Tp-e remained a significant predictor of SCD. In subjects with normal QTc, Tp-e remained significantly correlated with SCD [27]. Helmy et al. [28] observed a strong positive association between QTc dispersion and SS among patients with STEMI during the past six months. Bektas el al. [29] examined 172 patients who underwent coronary angiography for SAP retrospectively. Patients were grouped as patients with a low and high SS (<22 and > 22). Although the QTc and QT values were identical, the Tp-e in the high SYNTAX group 
was significantly higher $(p=0.045)$. Furthermore, both the Tp-e/QTc and Tp-e/QT ratios in the high SYNTAX group were higher ( $\mathrm{p}<0.001)$. However, in our study we found that QT duration, Tp-e interval and Tp-e/QTc and Tp-e/QT were similar between high and low SYNTAX groups and also were similar to normal coronary artery group as well. The lack of clinical value of such electrocardiographic parameters may be due to scarce of very severe or subtotal stenosis that may cause significant ischemia and ischemia induced arrhythmias. The number of patients presenting with arrythmia in stable angina pectoris not having necrosis or severe ischemia itself may be so rare and it may not be possible to detect significant ECG changes in such a small study group. Ipek et al. [30] stated that intervals of Tp-e and cTp-e, and ratio of Tp-e/QT were significantly higher among SAP patients with a high SS. The intervals of Tp-e and cTp-e, and ratio of Tpe/QT were associated prominently and moderately with SS $\quad(r=0.435 / p=0.005$, $r=0.395 / p=0.004$ and $r=0.348 / p=0.011$, respectively). On the other hand, they have not handled intervals of JT and JTc, and Tp-e/JT or Tp-e/JTc. Contrary to their results, in our study there were no substantial differences between high SS, low SS and normal coronary artery groups regarding Tp-e and JT intervals, and Tpe/QTc, Tp-e/JTc and Tp-e/JT.

One of the most major limitations of this analysis is the relatively small sample size. Additionally, another constraint is the manual calculation of non-computer-aided ECG measurements. In addition, classifying patients with methods such as myocardial perfusion scintigraphy or fractional flow reserve according to evidence of ischemia could reveal more reliable results. The study lacks follow up for the detection of arrhythmias and their association with ECG parameters.

\section{Conclusion}

Several surface ECG predictors of ventricular arrhythmias, including QTd, JT and Tp-e values and their ratios to JT and QT, are not significantly correlated with SYNTAX scoreassessed CAD severity.

Funding: The author(s) received no financial support for the research, authorship, and/or publication of this article.

Conflict of Interest: The authors declare that they have no conflict of interest.

Ethical statement: The study was approved by the Ethics committee of Bolu Abant Izzet Baysal University (Date and decision number: 2020/06)

\section{Open Access Statement}

This is an open access journal which means that all content is freely available without charge to the user or his/her institution under the terms of the Creative Commons Attribution NonCommercial License (http://creativecommons.org/licenses/bync/4.0). Users are allowed to read, download, copy, distribute, print, search, or link to the full texts of the articles, without asking prior permission from the publisher or the author.

\section{References}

[1]Nowbar AN, Gitto M, Howard JP, at al. Mortality From Ischemic Heart Disease. Circ Cardiovasc Qual Outcomes. 2019;12(6): e005375.

[2]Sara JD, Eleid MF, Gulati R, et al. Sudden Cardiac Death From the Perspective of Coronary Artery Disease. Mayo Clin Proc. 2014;89(12):1685-98.

[3]Priori SG, Blomström-Lundqvist C, Mazzanti A, et al. Task Force for the Management of Patients with Ventricular Arrhythmias and the Prevention of Sudden 
Cardiac Death of the European Society of Cardiology (ESC). Europace. 2015;17(11):1601-87.

[4]Engel G, Beckerman JG, Froelicher VF, et al. Electrocardiographic arrhythmia risk testing. Curr Probl Cardiol. 2004;29(7):36532.

[5]Turakhia M, Tseng ZH. Sudden cardiac death: epidemiology, mechanisms, and therapy. Curr Probl Cardiol. 2007;32(9):501-46.

[6]Castro-Torres Y, Carmona-Puerta R, Katholi RE. Ventricular repolarization markers for predicting malignant arrhythmias in clinical practice. World J Clin Cases. 2015;3(8):705-20.

[7]Crow RS, Hannan PJ, Folsom AR. Prognostic significance of corrected QT and corrected JT interval for incident coronary heart disease in a general population sample stratified by presence or absence of wide QRS complex: the ARIC Study with 13 years of follow-up. Circulation. 2003;108(16):1985-89.

[8]Gupta P, Patel C, Patel H et al. T(p-e)/QT ratio as an index of arrhythmogenesis. $\mathrm{J}$ Electrocardiol. 2008;41(6):567-74.

[9]Castro-Torres Y. Tpeak-Tend/QT: un nuevo predictor electrocardiográfico de muerte súbita cardíaca. Cardiocore. 2014;49(2):8687.

[10] Sianos G, Morel MA, Kappetein AP, et al. The SYNTAX score: an angiographic tool grading the complexity of coronary artery disease. Euro Intervention. 2005;1(2):21927.

[11] Valgimigli M, Serruys PW, Tsuchida K, et al. Cyphering the complexity of coronary artery disease using the syntax score to predict clinical outcome in patients with three-vessel lumen obstruction undergoing percutaneous coronary intervention. Am J Cardiol. 2007;99(8):1072-81.

[12]Lemesle G, Bonello L, de Labriolle A, et al. Prognostic value of the Syntax score in patients undergoing coronary artery bypass grafting for three-vessel coronary artery disease. Catheter Cardiovasc Interv. 2009;73(5):612-17.

[13]Capodanno D, Di Salvo ME, Cincotta G, et al. Usefulness of the SYNTAX score for predicting clinical outcome after percutaneous coronary intervention of unprotected left main coronary artery disease. Circ Cardiovasc Interv. 2009;2(4):302-8.

[14] Bazett HC. An analysis of the time relations of electrocardiograms. Heart. 1920;7:35370.

[15] Kolettis TM. In: Kibos A, Knight B, Essebag V, Fishberger S, Slevin M, Țintoiu I, editor. Ventricular Arrhythmias During Acute Myocardial Ischemia/Infarction: Mechanisms and Management. Cardiac Arrhythmias: Springer; 2014. p. 237-251.

[16]Di Diego JM, Antzelevitch C. Ischemic ventricular arrhythmias: Experimental models and their clinical relevance. Heart Rhythm. 2011;8(12):1963-68.

[17] Wei N, Tolkacheva EG. Interplay between ephaptic coupling and complex geometry of border zone during acute myocardial ischemia: Effect on arrhythmogeneity. Chaos: An Interdisciplinary Journal of Nonlinear Science. 2020;30(3):033111.

[18]Bernus O, Zemlin CW, Zaritsky RM, et al. Alternating conduction in the ischaemic border zone as precursor of reentrant arrhythmias: A simulation study. Europace. 2005;7(2):93-104.

[19]Wilde AA. Role of ATP-sensitive K+ channel current in ischemic arrhythmias. Cardiovasc Drugs Ther. 1993;7(3):521-26. 
[20]Monitillo F, Leone M, Rizzo C, et al. Ventricular repolarization measures for arrhythmic risk stratification. World $\mathbf{J}$ Cardiol. 2016;8(1):57-73.

[21] Kautzner J. QT Interval Measurements. Card Electrophysiol Rev. 2002;6(3):273-77.

[22] Spodick DH. Reduction of QT-interval imprecision and variance by measuring the JT interval. Am J Cardiol. 1992;70(1):62829.

[23] Antzelevitch C. T peak-Tend interval as an index of transmural dispersion of repolarization. Eur $\mathrm{J}$ Clin Invest. 2001;31(7):555-57.

[24] Antzelevitch C. Heterogeneity and cardiac arrhythmias: an overview. Heart Rhythm. 2007;4(7):964-72.

[25] Yilmaz R, Demirbag R, Gur M. The association of QT dispersion and QT dispersion ratio with extent and severity of coronary artery disease. Ann Noninvasive Electrocardiol. 2006;11(1):43-51.

[26] Panikkath R, Reinier K, Uy-Evanado A, et al. Prolonged Tpeak-to-tend interval on the resting ECG is associated with increased risk of sudden cardiac death. Circ Arrhythm Electrophysiol. 2011;4(4):441-47.

[27]Chugh SS, Reinier K, Singh $T$, et al. Determinants of prolonged QT interval and their contribution to sudden death risk in coronary artery disease: the Oregon Sudden Unexpected Death Study. Circulation. 2009;119(5):663-70.

[28] Helmy H, Abdel-Galeel A, Kishk YT, et al. Correlation of corrected QT dispersion with the severity of coronary artery disease detected by SYNTAX score in non-diabetic patients with STEMI. Egypt Heart J. 2017;69(2):111-17.

[29]Bektaş O. The Association Between Coronary Artery Disease Severity And Tp-e İnterval, Tp-e/qt Ratio Parameters Including
Fragmented Qrs. Am J Cardiol. 2018;121(8):e54.

[30] İpek E, Yolcu M, Yildırım E, et al. The Relationship Between Electrocardiographic Tp-e Interval and Gensini \& Syntax Scores in Patients with Stable Angina Pectoris. MN Kardiyoloji. 2016;23(3):126-31. 